\title{
Insecurity Of Imperfect Quantum Bit Seal
}

\author{
H. F. Chav* \\ Department of Physics, University of Hong Kong, Pokfulam Road, Hong Kong and \\ Center of Theoretical and Computational Physics, \\ University of Hong Kong, Pokfulam Road, Hong Kong
}

(Dated: November 21, 2018)

\begin{abstract}
Quantum bit seal is a way to encode a classical bit quantum mechanically so that everyone can obtain non-zero information on the value of the bit. Moreover, such an attempt should have a high chance of being detected by an authorized verifier. Surely, a reader looks for a way to get the maximum amount of information on the sealed bit and at the same time to minimize her chance of being caught. And a verifier picks a sealing scheme that maximizes his chance of detecting any measurement of the sealed bit. Here, I report a strategy that passes all measurement detection procedures at least half of the time for all quantum bit sealing schemes. This strategy also minimizes a reader's chance of being caught under a certain scheme. In this way, I extend the result of Bechmann-Pasquinucci et al. by proving that quantum seal is insecure in the case of imperfect sealed bit recovery.
\end{abstract}

PACS numbers: 03.67.Dd, 03.67.Hk, 89.20.Ff, 89.70.+c

Keywords: Information disturbance tradeoff, Post-modern quantum cryptography, Quantum seal

Introduction - We sometimes put an important document, such as a will, in an envelop with sealed wax so that others can open it only by breaking the wax. The intactness of the wax seal, therefore, shows that the document has not been opened. It is useful to extend the concept of physical wax seal to the digital world. Yet, no classical digital sealing scheme is unconditionally secure as one can, in principle, copy all the bits without being caught.

Recently, Bechmann-Pasquinucci explored the possibility of sealing a classical bit quantum mechanically. In her scheme, each bit of classical message is encoded as three qubits out of which one of them is erroneous. Using single qubit measurement along the standard basis plus the classical $[3,1,3]_{2}$ majority vote code, everyone can obtain the original classical bit with certainty. Moreover, an authorized verifier, who knows some extra information on the erroneous qubit, may check if someone has extracted the encoded classical bit with non-negligible probability 1]. Following a similar line, Chau proposed quantum bit seal based on quantum error correcting code that applied to quantum messages [2]. Recently, Singh and Srikanth [3] as well as He [4, 5] separately constructed quantum seals by extending the Bechmann-Pasquinucci protocol in different ways. In particular, He constructed a quantum bit string seal [5].

The above five quantum sealing schemes can be divided into two types. The first two are perfect quantum seals in the sense that a reader can obtain the classical message with certainty. The last three are imperfect for a reader cannot do so accurately.

The density matrices used to encode any two distinct classical messages in a perfect quantum seal must be orthogonal. Hence, one may devise a collective measurement to read out the sealed message without disturbing the encoded state. (A quantum computer may be re- quired to perform the collective measurement though.) This is precisely the idea used by Bechmann-Pasquinucci et al. to prove the insecurity of all perfect quantum seals [6]. However, their proof does not apply to imperfect seals. Therefore, it is instructive to study the security of imperfect seals. Recently, He analyzed the security of imperfect quantum bit seals and proved certain bounds regarding the information gain and the measurement detection probability 7]. However, his bound is not tight.

In this Letter, I study the relation between the information on the sealed bit obtained by a reader and the probability of measurement detection by a verifier. In particular, I find a cheating strategy that obtains nonzero amount of information on the sealed bit but escapes detection at least half of the time for all quantum bit sealing schemes. Moreover, this strategy is optimal for a certain bit sealing scheme in the sense that no strategy with the same power of distinguishing the two sealed bits has a higher chance of avoiding measurement detection under that scheme. (Implementing this cheating strategy may require a quantum computer.) Consequently, I conclude that all imperfect quantum bit seals are insecure.

Quantum Bit Seal - A quantum bit seal is a method for Alice to encode a bit in such a way that any member of the public, say Bob, can recover the original bit with probability greater than a half. Moreover, any recovery process must disturb the encoded state so that the intactness of the state plays the role of a wax seal. By checking its intactness, an authorized verifier may correctly detect any measurement of the value of the sealed bit with a certain non-zero probability. Without loss of generality, one may assume that the encoded state $i$ Alice prepared is a pure state given by

$$
\left|\tilde{\psi}_{i}\right\rangle=\sum_{j} \lambda_{i j}\left|\psi_{i j}\right\rangle_{B} \otimes\left|\phi_{j}\right\rangle_{A}
$$


for $i=0,1$, where $\left|\psi_{i j}\right\rangle$ 's are normalized states that are not necessarily mutually orthogonal, and $\left|\phi_{j}\right\rangle$ 's are orthonormal states. (If the states prepared by Alice are mixed, she can always purify them. More importantly, the use of pure states increases the verifier's chance to detect a cheating Bob.) She makes the particles labeled by the subscript "B" publicly accessible, and keeps those labeled by the subscript "A" for authorized verifiers only. So, from Bob's point of view, the sealed state is either $\rho_{0}$ or $\rho_{1}$, where

$$
\rho_{i}=\sum_{j}\left|\lambda_{i j}\right|^{2}\left|\psi_{i j}\right\rangle\left\langle\psi_{i j}\right|
$$

The maximum achievable classical $L_{1}$ distance between probability distributions arising from measurements performed on $\rho_{0}$ and $\rho_{1}$ is equal to the trace distance between the two density matrices [8]

$$
q_{\max }=D\left(\rho_{0}, \rho_{1}\right) \equiv \frac{1}{2} \operatorname{Tr}\left|\rho_{0}-\rho_{1}\right| .
$$

The higher the value of $q_{\max }$, the higher the chance for Bob to correctly extract the sealed bit. In fact, a quantum bit seal is perfect if $q_{\max }=1$; and it is imperfect if $0 \leq q_{\max }<1$. (Actually, the case of $q_{\max }=0$ is not a quantum bit seal at all for it gives no information on the original state.)

To gain information on the sealed state, Bob performs a positive operator-valued measure (POVM) measurement $\mathcal{E}$ on the publicly accessible state. The classical $L_{1}$ distance between probability distributions arising from the measurement $\mathcal{E}$ on $\rho_{0}$ and $\rho_{1}$ equals $q \equiv$ $D\left(\mathcal{E}\left(\rho_{0}\right), \mathcal{E}\left(\rho_{1}\right)\right)$. From Alice's point of view, the entire entangled state becomes $\mathcal{E} \otimes I\left(\left|\tilde{\psi}_{i}\right\rangle\left\langle\tilde{\psi}_{i}\right|\right) \equiv \tilde{\mathcal{E}}\left(\left|\tilde{\psi}_{i}\right\rangle\left\langle\tilde{\psi}_{i}\right|\right)$. Amongst all $\mathcal{E}$ whose measurement results on $\rho_{0}$ and $\rho_{1}$ have the same classical $L_{1}$ distance $q \in\left[0, q_{\max }\right]$, Bob would like to pick the one that minimizes the chance of being detected by a verifier. Since it is equally likely for the sealed bit to be 0 or 1 and the probability of catching the reader Bob is at most equal to the fidelity of the resultant state as $\left|\tilde{\psi}_{i}\right\rangle$, Bob's aim is equivalent to finding a quantum operation $\tilde{\mathcal{E}}=\mathcal{E} \otimes I$ that maximizes the average fidelity

$$
\bar{F}=\frac{1}{2} \sum_{i=0}^{1}\left\langle\tilde{\psi}_{i}\left|\tilde{\mathcal{E}}\left(\left|\tilde{\psi}_{i}\right\rangle\left\langle\tilde{\psi}_{i}\right|\right)\right| \tilde{\psi}_{i}\right\rangle \equiv\left\langle\operatorname{Tr}\left[\tilde{\mathcal{E}}\left(\tilde{\rho}_{i}\right) \tilde{\rho}_{i}\right]\right\rangle_{i} .
$$

On the other hand, Alice has the freedom to pick the sealing scheme. Of course, she would like to choose the one that minimizes $\bar{F}$. Consequently, the average fidelity for the optimal cheating strategy $\min _{\text {Alice }} \max _{\mathrm{Bob}} \bar{F}\left(q, q_{\max }\right)$ is found by first taking the maximum over all possible POVM measurements $\mathcal{E}$ used by Bob with $q=$ $D\left(\mathcal{E} \otimes I\left(\tilde{\rho}_{0}\right), \mathcal{E} \otimes I\left(\tilde{\rho}_{1}\right)\right)$ for a given sealing scheme, and then by taking the minimum over all quantum bit seals with trace distance $q_{\max }$ chosen by Alice. Here, I prove that

$$
\min _{\text {Alice }} \max _{\mathrm{Bob}} \bar{F}\left(q, q_{\max }\right)=\frac{1+q_{\max }^{2}}{2}+\frac{1-q_{\max }^{2}}{2}\left(1-\frac{q^{2}}{q_{\max }^{2}}\right)^{\frac{1}{2}}
$$

for all $0<q \leq q_{\max }$ and that

$$
\min _{\text {Alice Bob }} \max _{\text {Bob }} \bar{F}\left(0, q_{\max }\right)=1 \text {. }
$$

The Optimal Cheating Strategy - Let me introduce a few notations before reporting the optimal cheating strategy that works for $q>0$. (Note that this strategy is optimal in the sense stated in the above paragraph. That is to say, this is the best strategy for Bob to work against the most stringent sealing scheme devised by Alice. It is possible, however, to find a strategy with a higher value of average fidelity if Alice uses a less stringent scheme.) I write $\rho_{0}-\rho_{1}=Q_{0}-Q_{1}$, where $Q_{i}$ 's are positive operators with orthogonal support. Suppose $\Pi_{0}$ satisfying $\Pi_{0} Q_{0}=Q_{0}$ is a projector whose support is orthogonal to that of $Q_{1}$, and let $\Pi_{1}=I-\Pi_{0}$ be its complementary projector. (Clearly, these projectors exist and are non-zero if and only if $q_{\max }>0$. In addition, these non-zero projectors are uniquely determined on the support of $\rho_{0}+\rho_{1}$ if and only if $\rho_{0}-\rho_{1}$ and $\rho_{0}+\rho_{1}$ have a common support.) Then, the classical $L_{1}$ distance between probability distributions arising from the POVM measurement with measurement operators $\left\{\Pi_{0}, \Pi_{1}\right\}$ on $\rho_{0}$ and $\rho_{1}$ equals $q_{\max }[8,[9]$. By denoting

$$
a=\left\langle\tilde{\psi}_{0}\left|\Pi_{0} \otimes I\right| \tilde{\psi}_{0}\right\rangle,
$$

one has

$$
\begin{aligned}
\left\langle\tilde{\psi}_{0}\left|\Pi_{1} \otimes I\right| \tilde{\psi}_{0}\right\rangle & =1-a, \\
\left\langle\tilde{\psi}_{1}\left|\Pi_{0} \otimes I\right| \tilde{\psi}_{1}\right\rangle & =a-q_{\max }
\end{aligned}
$$

and

$$
\left\langle\tilde{\psi}_{1}\left|\Pi_{1} \otimes I\right| \tilde{\psi}_{1}\right\rangle=1-a+q_{\max } .
$$

Clearly, $q_{\max } \leq a \leq 1$.

For any fixed $0<q \leq q_{\max }$, let

$$
M_{0}=\left[\frac{1}{2}\left(1+\frac{q}{q_{\max }}\right)\right]^{\frac{1}{2}} \Pi_{0}+\left[\frac{1}{2}\left(1-\frac{q}{q_{\max }}\right)\right]^{\frac{1}{2}} \Pi_{1}
$$

and

$$
M_{1}=\left[\frac{1}{2}\left(1-\frac{q}{q_{\max }}\right)\right]^{\frac{1}{2}} \Pi_{0}+\left[\frac{1}{2}\left(1+\frac{q}{q_{\max }}\right)\right]^{\frac{1}{2}} \Pi_{1} .
$$

Then $M_{0}^{\dagger} M_{0}+M_{1}^{\dagger} M_{1}=I$. So, the operation $\mathcal{E}_{q, q_{\max }}$ given by

$$
\mathcal{E}_{q, q_{\max }}\left(\rho_{i}\right)=M_{0} \rho_{i} M_{0}^{\dagger}+M_{1} \rho_{i} M_{1}^{\dagger}
$$


is a POVM measurement with $D\left(\mathcal{E}_{q, q_{\max }}\left(\rho_{0}\right), \mathcal{E}_{q, q_{\max }}\left(\rho_{1}\right)\right)$ $=q$. Bob applies this POVM measurement to the publicly accessible quantum particles and deduces the value of the sealed bit as $i$ if the measurement outcome is $i$. (Bob may require a quantum computer in order to implement this measurement.)

From Eqs. (7)-(12), the probability of correctly determine the sealed bit equals

$$
\operatorname{Pr}=\left\langle\left\langle\tilde{\psi}_{i}\left|M_{i}^{\dagger} M_{i}\right| \tilde{\psi}_{i}\right\rangle\right\rangle_{i}=\frac{1+q}{2}
$$

Similarly, the average fidelity of the resultant state caused by this cheating strategy is given by

$$
\begin{aligned}
\bar{F}(q)= & \frac{1}{2} \sum_{i, j=0}^{1}\left|\left\langle\tilde{\psi}_{i}\left|M_{j} \otimes I\right| \tilde{\psi}_{i}\right\rangle\right|^{2} \\
= & \frac{1}{2} \sum_{i=0}^{1}\left[\sum_{j=0}^{1} \operatorname{Tr}\left(\Pi_{j} \otimes I \tilde{\rho}_{i} \Pi_{j} \otimes I \tilde{\rho}_{i}\right)\right. \\
& \left.+2\left(1-\frac{q^{2}}{q_{\max }^{2}}\right)^{1 / 2} \operatorname{Tr}\left(\Pi_{0} \otimes I \tilde{\rho}_{i} \Pi_{1} \otimes I \tilde{\rho}_{i}\right)\right] \\
= & (1-2 a)\left(1+q_{\max }\right)+2 a^{2}+q_{\max }^{2}-\left[2 a^{2}\right. \\
& \left.+\left(q_{\max }-2 a\right)\left(1+q_{\max }\right)\right]\left(1-\frac{q^{2}}{q_{\max }^{2}}\right)^{\frac{1}{2}}
\end{aligned}
$$

Note that $\bar{F}(q)$ attains its minimum value when $a=$ $\left(1+q_{\max }\right) / 2$. More importantly, from the construction of $\Pi_{i}$ 's, Alice may force $a$ to take on the value $\left(1+q_{\max }\right) / 2$ by choosing $\left|\tilde{\psi}_{i}\right\rangle$ 's in such a way that the supports of $\rho_{0}-$ $\rho_{1}$ and $\rho_{0}+\rho_{1}$ agree and that $\left\langle\tilde{\psi}_{0}\left|\Pi_{0}\right| \tilde{\psi}_{0}\right\rangle=\left(1+q_{\max }\right) / 2$. (Such $\left|\tilde{\psi}_{i}\right\rangle$ 's certainly exist. One possible choice is $\left|\tilde{\psi}_{i}\right\rangle=$ $\left\{\left[1+(-1)^{i} q_{\max }\right] / 2\right\}^{1 / 2}|0\rangle_{B}+\left\{\left[1-(-1)^{i} q_{\max }\right] / 2\right\}^{1 / 2}|1\rangle_{B}$ for $i=0,1$.) Once Alice has picked such $\left|\tilde{\psi}_{i}\right\rangle$ 's, the average fidelity of the resultant state after applying the POVM measurement $\mathcal{E}_{q, q_{\max }}$ is

$$
\bar{F}(q)=\frac{1+q_{\max }^{2}}{2}+\frac{1-q_{\max }^{2}}{2}\left(1-\frac{q^{2}}{q_{\max }^{2}}\right)^{\frac{1}{2}} .
$$

This $\bar{F}(q)$ sets the lower bound for the value of $\min _{\text {Alice }} \max _{\text {Bob }} \bar{F}\left(q, q_{\max }\right)$ in the case of $q>0$.

The Proof Of Optimality - I prove the optimality of the cheating strategy $\mathcal{E}_{q, q_{\max }}$ by explicitly constructing a quantum bit seal whose value of $\bar{F}(q)$ is upper bounded by the right hand side of Eq. (16) for any cheating strategy used by Bob. I claim that

$$
\begin{aligned}
\left|\tilde{\psi}_{i}\right\rangle= & \frac{\sqrt{1-q_{\max }}}{2}\left\{\left[|0\rangle_{B}+(-1)^{i}|1\rangle_{B}\right] \otimes|0\rangle_{A}\right. \\
& \left.+\left[|0\rangle_{B}-(-1)^{i}|1\rangle_{B}\right] \otimes|1\rangle_{A}\right\} \\
& +q_{\max }^{1 / 2}|i\rangle_{B} \otimes|2\rangle_{A}
\end{aligned}
$$

for $i=0,1$ is such a scheme.
Clearly, $\rho_{i}=\operatorname{Tr}_{A}\left(\left|\tilde{\psi}_{i}\right\rangle\left\langle\tilde{\psi}_{i}\right|\right)=\operatorname{diag}\left(\left[1+(-1)^{i} q_{\max }\right] / 2\right.$, $\left.\left[1-(-1)^{i} q_{\max }\right] / 2\right)$. Thus, $D\left(\rho_{0}, \rho_{1}\right)=q_{\max }$.

Recall that the most general measurement strategy for Bob is to perform a POVM measurement with measurement operators $N_{j}$ 's, where

$$
N_{j}=\left[\begin{array}{ll}
\alpha_{j} & \beta_{j} \\
\gamma_{j} & \delta_{j}
\end{array}\right]
$$

To qualify as a POVM measurement whose measurement results on $\rho_{0}$ and $\rho_{1}$ give probability distributions with classical $L_{1}$ distance $q$, one requires

$$
\begin{gathered}
\sum_{j}\left(\left|\alpha_{j}\right|^{2}+\left|\gamma_{j}\right|^{2}\right)=1=\sum_{j}\left(\left|\beta_{j}\right|^{2}+\left|\delta_{j}\right|^{2}\right) \\
\sum_{j}\left(\alpha_{j} \bar{\beta}_{j}+\gamma_{j} \bar{\delta}_{j}\right)=0
\end{gathered}
$$

and

$$
\left.q_{\max } \sum_{j}|| \alpha_{j}\right|^{2}+\left|\gamma_{j}\right|^{2}-\left|\beta_{j}\right|^{2}-\left|\delta_{j}\right|^{2} \mid=2 q .
$$

The average fidelity of the state after applying $\mathcal{E}_{q, q_{\max }}$ equals

$$
\begin{aligned}
\bar{F}(q)= & \frac{1+q_{\max }^{2}}{4} \sum_{j}\left(\left|\alpha_{j}\right|^{2}+\left|\delta_{j}\right|^{2}\right) \\
& +\frac{1-q_{\max }^{2}}{4} \sum_{j}\left(\alpha_{j} \bar{\delta}_{j}+\bar{\alpha}_{j} \delta_{j}\right) .
\end{aligned}
$$

The constraint in Eq. (19) implies that

$$
\sum_{j}\left(\left|\alpha_{j}\right|^{2}+\left|\delta_{j}\right|^{2}\right) \leq 2
$$

And by constrained maximization, one concludes that

$$
\sum_{j}\left(\alpha_{j} \bar{\delta}_{j}+\bar{\alpha}_{j} \delta_{j}\right) \leq 2\left(1-\frac{q^{2}}{q_{\max }^{2}}\right)^{\frac{1}{2}}
$$

Consequently, $\bar{F}(q)$ for this scheme is less than or equal to the right hand side of Eq. (16). Therefore, the optimality of $\mathcal{E}_{q, q_{\max }}$ together with the validity of Eq. (5) are proven.

It remains to consider the case of $q=0$. In this case, Bob simply applies the identity operator to the state. Hence, $\min _{\text {Alice }} \max _{\text {Bob }} \bar{F}\left(0, q_{\max }\right)=1$. Note that the positive operators $Q_{0}$ and $Q_{1}$ are non-zero if and only if $q_{\max }>0$. The sudden change in the dimensions of $Q_{i}$ 's is probably the reason why $\min _{\text {Alice }} \max _{\mathrm{Bob}} \bar{F}\left(q, q_{\max }\right)$ is discontinuous at $(0,0)$.

Discussions - In short, I have studied the relation between the information gain on the sealed qubit and the probability of sealed bit measurement detection. In 
particular, I find a cheating strategy $\mathcal{E}_{q, q_{\max }}$ whose probability of passing any measurement detection procedure is greater than or equal to the right hand side of Eq. (16). Since this probability is at least $1 / 2$, I conclude that both perfect and imperfect quantum bit seals are insecure. This strategy reduces to the one used by BechmannPasquinucci et al. 6 in the case of a perfect seal. More importantly, the cheating strategy $\mathcal{E}_{q, q_{\max }}$ is optimal in the sense that it has the least possible chance of being detected by a verifier for the most stringent quantum bit sealing scheme. Although all quantum states presented in this Letter live in finite dimensional Hilbert spaces, the arguments used in the proof are completely general and are applicable also to states living in infinite dimensional Hilbert spaces.

At least two lines of followup researches are worth to conduct. One line is to investigate the security of imperfect quantum bit string seal. Although one may apply the cheating strategy reported above to correctly read out a single bit in any quantum bit string seal with a small chance of being caught, the possibility (or impossibility) of correctly determining the values of a large proportion of bits without being caught remains unclear. The other line of research is to look for a quantum bit sealing scheme whose security is based on computational (such as the inefficiency in computing $Q_{0}$ and $Q_{1}$ ) or hardware (such as the hardness of building a quantum computer) assumptions.

Lastly, I remark that there is an irreparable gap in the "security proof" of the quantum seal in my earlier manuscript in Ref. [2] - there is no way for Alice to force Bob to measure the publicly accessible qubits individually.

Acknowledgments - This work is supported by the RGC grant HKU 7010/04P of the HKSAR Government.

* Electronic address: hfchau@hkusua.hku.hk

[1] H. Bechmann-Pasquinucci, Int. J. Quant. Inform. 1, 217 (2003).

[2] H. F. Chau, Sealing quantum message by quantum code, quant-ph/0308146.

[3] S. K. Singh and R. Srikanth, Physica Scripta 71, 433 (2005).

[4] G.-P. He, Quantum secret sharing, hiding and sealing of classical data against collective measurement, quant$\mathrm{ph} / 0502091 \mathrm{v} 1$.

[5] G.-P. He, Quantum bit string sealing, quantph/0502091v3.

[6] H. Bechmann-Pasquinucci, G. M. D'Ariano, and C. Macchiavello, Int. J. Quant. Inform. 3, 435 (2005).

[7] G.-P. He, Phys. Rev. A 71, 054304 (2005).

[8] M. A. Nielsen and I. L. Chuang, Quantum Computation and Quantum Information (CUP, Cambridge, 2000), chap. 9.

[9] C. W. Helstrom, Quantum Detection and Estimation Theory (Academic Press, New York, 1976), chap. IV. 